\title{
ARTICLE Metaplasticity contributes to memory formation in the hippocampus
}

\author{
Ana P. Crestani ${ }^{1}$, Jamie N. Krueger ${ }^{2}$, Eden V. Barragan ${ }^{2}$, Yuki Nakazawa ${ }^{3}$, Sonya E. Nemes ${ }^{4}$, Jorge A. Quillfeldt ${ }^{5}$,
} John A. Gray (D) $^{3,6}$ and Brian J. Wiltgen ${ }^{3,7}$

Prior learning can modify the plasticity mechanisms that are used to encode new information. For example, NMDA receptor (NMDAR) activation is typically required for new spatial and contextual learning in the hippocampus. However, once animals have acquired this information, they can learn new tasks even if NMDARs are blocked. This finding suggests that behavioral training alters cellular plasticity mechanisms such that NMDARs are not required for subsequent learning. The mechanisms that mediate this change are currently unknown. To address this issue, we tested the idea that changes in intrinsic excitability (induced by learning) facilitate the encoding of new memories via metabotropic glutamate receptor (mGluR) activation. Consistent with this hypothesis, hippocampal neurons exhibited increases in intrinsic excitability after learning that lasted for several days. This increase was selective and only observed in neurons that were activated by the learning event. When animals were trained on a new task during this period, excitable neurons were reactivated and memory formation required the activation of mGluRs instead of NMDARs. These data suggest that increases in intrinsic excitability may serve as a metaplastic mechanism for memory formation.

Neuropsychopharmacology (2019) 44:408-414; https://doi.org/10.1038/s41386-018-0096-7

\section{INTRODUCTION}

The way we learn is influenced by our past. Previous experiences can impede new learning as in the case of proactive interference $[1,2]$, or they can enhance learning by providing a schema into which new information can be rapidly incorporated $[3,4]$. While these phenomena are well established at the psychological level, much less is known about the impact of experience on the cellular mechanisms of learning and memory. This is true, in part, because most experiments are done with laboratory animals that have little to no real-world experience. It is possible that these naïve animals encode information using different mechanisms than more experienced subjects. If so, contemporary ideas about memory may be limited to a restricted set of conditions that rarely exist in nature. The current experiments examined this possibility by studying learning mechanisms in animals with prior behavioral experience.

Experience is known to influence the role of NMDA receptors (NMDARs) in learning and memory. For example, rats with no experience in the water maze are severely impaired when NMDARs are blocked in the hippocampus during training. This same manipulation has no effect, however, if animals are pretrained on the task [5]. This is true even when pre-training occurs in a different maze with a distinct set of spatial cues [6]. Similar effects have been observed for context fear conditioning and olfactory learning [7-11]. In each case, initial learning requires NMDAR activation while subsequent learning does not.
While it is known that prior experience alters the substrates of memory, it is unclear how this occurs. One possibility is that learning induces non-synaptic changes (like increases in intrinsic excitability) that alter the way neurons encode subsequent information [12, 13]. Increased excitability has been observed in the hippocampus after learning and can last for several days depending on the salience of the initial experience [13-15]. If an animal acquires new information during this period, it could be rapidly encoded by excitable neurons via NMDAR-independent plasticity mechanisms $[7,13]$. To examine this idea, we tagged hippocampal neurons that were activated by context fear conditioning and determined if they were more excitable than non-tagged cells 2 days later. We also determined if tagged cells were reactivated when animals encoded a second memory 2 days after the first.

Some forms of hippocampal long-term potentiation (LTP) do not require NMDAR activation. These include mossy fiber LTP in CA3 and high-frequency LTP $(200 \mathrm{~Hz})$ in CA1. Interestingly, in both cases, synaptic strengthening requires the activation of metabotropic glutamate receptors (mGluRs) [16, 17]. Consequently, we determined if mGluRs contribute to NMDAR-independent learning. To do this, mice underwent fear conditioning in two distinct contexts in sequence (A followed by $B$ ). Our previous work showed that memory for context $A$ requires NMDAR activation while memory for context $B$ does not. To examine the role of mGluRs, we infused the group I/II antagonist MCPG into the hippocampus prior to learning in A or B.

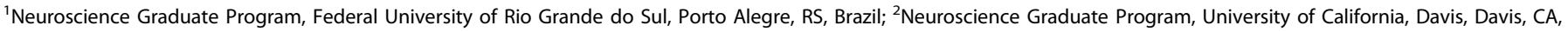

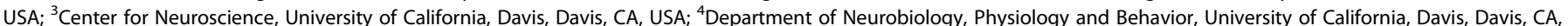

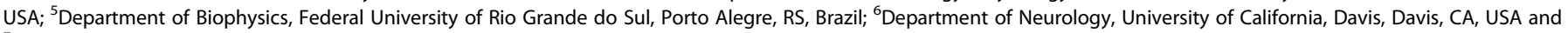
${ }^{7}$ Department of Psychology, University of California, Davis, Davis, CA, USA

Correspondence: Brian J. Wiltgen (bjwiltgen@ucdavis.edu)

These authors contributed equally: Ana P. Crestani, Jamie N. Krueger
}

Received: 16 February 2018 Revised: 4 May 2018 Accepted: 11 May 2018

Published online: 16 May 2018 


\section{MATERIALS AND METHODS}

Animals

Male B6/129 F1 hybrid mice, 8-11 weeks old, weighing $20-35 \mathrm{~g}$ (Taconic) were used for drug microinfusion experiments. Reactivation and electrophysiology experiments were performed using TetTag transgenic mice. These mice express H2B-GFP under the control of the c-Fos promoter. In these mice, activation of the $\mathrm{C}$ Fos promoter during learning leads to the expression of H2B-GFP in active neurons [18]. For reactivation and electrophysiology experiments, mice were removed from doxycycline $(40 \mathrm{mg} / \mathrm{kg})$ for 2 days prior to fear conditioning. Immediately following training, animals were put on high doxycycline $(1 \mathrm{~g} / \mathrm{kg})$ for 1 day, then returned to low doxycycline for the remainder of the experiment. Mice were single housed in plastic cages under a $12 \mathrm{~h}$ light/dark cycle and at constant temperature of $24 \pm 1{ }^{\circ} \mathrm{C}$, with water and food ad libitum. Behavioral tests and recordings were performed during the light phase of the cycle. All experiments were carried out in accordance with the National Institutes of Health guidelines and were approved by the UC Davis Institutional Animal Care and Use Committee (IACUC).

\section{Electrophysiology}

For whole-cell recordings, 8- to 9-week-old TetTag mice were anesthetized with isoflurane and transcardially perfused with icecold carbogen $\left(95 \% \mathrm{O}_{2} / 5 \% \mathrm{CO}_{2}\right)$-bubbled NMDG-based cutting solution [19] containing (in mM): $93 \mathrm{NMDG}, 2.5 \mathrm{KCl}, 1.25 \mathrm{NaH}_{2} \mathrm{PO}_{4}$, $30 \mathrm{NaHCO}_{3}, 20$ HEPES, 25 glucose, 5 sodium ascorbate, 2 thiourea, 3 sodium pyruvate, $10 \mathrm{MgSO}_{4}, 0.5 \mathrm{CaCl}_{2}$ (titrated to $\mathrm{pH} 7.3-7.4$ with concentrated $\mathrm{HCl}$; osmolarity of 300-310 mOsm). Acute transverse slices $(300 \mu \mathrm{m})$ of dorsal hippocampus were cut on a Leica VT1200 vibratome (Buffalo Grove, IL) in the ice-cold and oxygenated NMDG cutting solution described above, then transferred to an incubation chamber containing the same NMDG cutting solution for $15 \mathrm{~min}$ at $34^{\circ} \mathrm{C}$. Before recording, slices were recovered for at least $45 \mathrm{~min}$ then stored submerged for up to $5 \mathrm{~h}$ in room temperature oxygenated artificial cerebrospinal fluid containing (in mM): $119 \mathrm{NaCl}, 26.2 \mathrm{NaHCO}_{3}, 11$ glucose, $2.5 \mathrm{KCl}$, $1 \mathrm{NaH}_{2} \mathrm{PO}_{4}, 2.5 \mathrm{CaCl}_{2}$, and $1.3 \mathrm{MgSO}_{4}$. Whole-cell current clamp recordings were obtained from CA1 pyramidal neurons under visual guidance (DIC/infrared optics). $\mathrm{GFP}^{+}$neurons were identified by epifluorescence microscopy. Whenever possible, neighboring $\mathrm{GFP}^{+}$and $\mathrm{GFP}^{-}$neurons were recorded simultaneously. In the current study, there were four pairs of simultaneously recorded neurons, with the remaining $N$ representing recordings when one of the two patches were unsuccessful (additional $n=4$ for GFP and $n=5$ for $\mathrm{GFP}^{+}$neurons). For all the recordings, borosilicate glass pipettes were fabricated with resistances of 4-6 M $\Omega$. Pipettes were filled with the following intracellular solution (in $\mathrm{mM}$ ): $135 \mathrm{~K}^{+}$ gluconate, $5 \mathrm{NaCl}, 2 \mathrm{MgCl}_{2}, 10$ HEPES, 0.6 EGTA, $4 \mathrm{NaATP}, 0.4$ NaGTP, pH 7.3, 290 mOsm. Recordings were collected with a Multiclamp 700B amplifier (Molecular Devices), filtered at $2 \mathrm{kHz}$, digitized at $10 \mathrm{kHz}$, and data analyzed using pClamp 10 software (Molecular Devices). Series resistance was monitored and cells in which series resistance varied by more than $20 \%$ during a recording were discarded. Frequency-current relationships for evoked firing were determined by injecting $500 \mathrm{~ms}$ current steps with amplitudes increasing by $20 \mathrm{pA}$, from 60 to $180 \mathrm{pA}$ from the resting potential. Liquid junction potentials were not corrected.

\section{Stereotaxic surgery}

Mice were anesthetized with isoflurane and mounted in a stereotaxic apparatus (David Kopf Instruments, Tujunga, CA, USA). Guide cannulae (22 gauge; Plastics One, Roanoke, VA) were inserted bilaterally into the dorsal hippocampus (anteriorposterior [AP] $-2.0 \mathrm{~mm}$ from bregma, mediolateral $[\mathrm{ML}] \pm 1.5 \mathrm{~mm}$, dorsoventral $[D V]-1.0 \mathrm{~mm}$. The cannulae were affixed to the skull using C\&B Metabond and a dental acrylic headcap.
Behavioral procedure

Prior to contextual fear conditioning, mice were handled 2 min a day for 6-7 days. The chamber used for fear conditioning in these experiments was described previously $[20,21]$. The chamber consisted of a stainless steel grid floor and side walls, as well as a Plexiglass door. Overhead LED lighting provided broad spectrum or near infrared light, and a high speed monochrome video camera (Med Associates) captured animals' movement during the training/testing sessions.

Mice underwent fear conditioning in two contexts: context $A$ followed by context B. Different visual, auditory, and olfactory cues were used to maximize distinction between contexts. Context $A$ had a level grid floor with visible lights and a fan turned on. Prior to conditioning, the chamber was cleaned with $70 \%$ ethanol. Context $B$ had a black plastic triangular tent inserted inside the chamber, red lights on with no visible lights and no fan. The level grid floor was replaced with a staggered grid floor and the chamber was cleaned with Saniwipes (Nice-Pak Products, Inc.).

The conditioning sessions were separated by 2 days. Memory was tested 1 day after training by returning mice to the conditioning context ( $\mathrm{A}$ or $\mathrm{B}$ ) for $5 \mathrm{~min}$ and measuring the freezing response. During training, mice were allowed to explore the conditioning context ( $\mathrm{A}$ or $\mathrm{B}$ ) for $3 \mathrm{~min}$ prior to the delivery of three $0.4 \mathrm{~mA} / 2 \mathrm{~s}$ footshocks with an inter-trial interval of $30 \mathrm{~s}$. The animals remained 30 additional seconds after the final shock, for a total of $276 \mathrm{~s}$ in the training context. During testing, mice were returned to the training chamber for $5 \mathrm{~min}(300 \mathrm{~s})$. The automated Video Freeze System (Med Associates) was used to quantify freezing, as described previously [22].

\section{Microinfusion}

Fifteen minutes prior to training (in context $A$ or context $B$ ), bilateral infusions of $0.5 \mu \mathrm{L}(0.1 \mu \mathrm{L} / \mathrm{min})$ were administered using $10 \mu \mathrm{L}$ Hamilton syringes controlled by a two-syringe micropump (Harvard Apparatus). The 28-gauge injection cannula projected an additional $1 \mathrm{~mm}$ from the tip of the guide cannula into the target region. Animals moved freely in an empty cage during the infusion procedure. The injectors were left in place for two additional minutes following infusion to allow drug diffusion. Mice were returned to their homecages until testing.

\section{Drugs}

APV or RS-MCPG (both from Tocris Bioscience) were used to block NMDAR and mGluR, respectively. The NMDAR antagonist APV (2.5 $\mu \mathrm{g} / \mu \mathrm{L})$ and mGluR antagonist MCPG $(1 \mu \mathrm{M})$ were dissolved in sterile saline. Saline served as the vehicle.

\section{Histology}

Histological verification of cannulae placements was performed at the end of behavioral testing. Mice were perfused transcardially with ice-cold phosphate-buffered saline (PBS) $\left(10 \mathrm{mM} \mathrm{PO}_{4}{ }^{3-}, 137\right.$ $\mathrm{mM} \mathrm{NaCl}$, and $2.7 \mathrm{mM} \mathrm{KCl}$ ) followed by $4 \%$ paraformaldehyde (PFA). Brains were post-fixed in 4\% PFA for 24-h prior to sectioning. Coronal sections $(40 \mu \mathrm{m})$ were cut on a vibratome and mounted on glass microscope slides. After drying, the sections were stained with cresyl violet to identify neuronal cell bodies. Cannulae placements were verified by visual inspection of the stained sections reconstructed on the mouse brain atlas $[22,36]$. We did not exclude any animals because of misplaced cannulae.

Immunohistochemistry

Immunohistochemistry for c-Fos was performed at the end of behavioral testing. Coronal sections ( $40 \mu \mathrm{m}$, dorsal hippocampus) were washed three times in $1 \times \mathrm{PBS}\left(10 \mathrm{mM} \mathrm{PO}_{4}{ }^{3-}, 137 \mathrm{mM} \mathrm{NaCl}\right.$, and $2.7 \mathrm{mM} \mathrm{KCl}$ ) before blocking buffer incubation and after all the antibodies and counterstaining steps. Slices were then blocked in donkey blocking buffer (DBB, $1 \mathrm{~mL}$ donkey serum, 
$100 \mu \mathrm{L}$ Triton-X, $50 \mathrm{~mL} 1 \times$ PBS) for $15 \mathrm{~min}$. Slices were placed overnight at $4{ }^{\circ} \mathrm{C}$ in 1:1000 rabbit anti c-Fos (Millipore) in DBB. Following first incubation, slices were placed in 1:500 donkey antirabbit biotynlated IGG (Jackson) for $1 \mathrm{~h}$ and then placed in 1:500 CY5 (Fisher) for $45 \mathrm{~min}$. Incubation in 1:10,000 DAPI for $10 \mathrm{~min}$ was performed as a counterstain. Slices were washed three times in $1 \times$ PBS and mounted onto Superfrost slides (Fisher). Slides were coverslipped with Vectashield antifade mounting media (Vector Labs).

\section{Microscopy and cell counting}

Slides were scanned using an Olympus Slide Scanner (Olympus). Images were cropped to proximal CA1 of the dorsal hippocampus. Cell counts were performed on three slices ( 6 hemispheres) from each animal. ImageJ was used to measure number of cells using volumetric analysis (Object Counter 3D) in the sample. GFP and cFos + cells were counted using the multi-point tool.

\section{Statistical analyses}

Evoked firing rates were analyzed using two-way ANOVA correcting for multiple comparisons with Sidak's correction. Resting membrane potential was analyzed using a two-tailed unpaired $t$-test. Statistical significance for analyses was set a priori at $p<0.05$. Freezing differences between groups were analyzed with two-way repeated measures ANOVAs and/or two-tailed unpaired $t$-tests. Percentages of GFP and c-Fos were analyzed with one-way repeated measures ANOVA and post-hoc comparisons were made using Tukey's test. Reactivation (GFP + c-Fos) differences were analyzed with two-tailed paired $t$-tests. All data are presented as mean \pm SEM.

\section{RESULTS}

Hippocampal neurons become more excitable after context fear learning

To determine if learning increases excitability, we patched and recorded from CA1 neurons after context fear conditioning (Fig. 1a). The long-lasting fluorescent protein H2B-GFP was used to identify cells that were active during training. Expression of this protein was controlled by the c-Fos promoter and suppressed by doxycycline as previously described [20, 21]. Doxycycline chow was removed 2 days prior to fear conditioning (to permit tagging) and reintroduced immediately after learning. Two days later, GFPpositive and GFP-negative cells were patched in CA1 and excitability was examined (Fig. 1b). We found a significant increase in intrinsic excitability (slope of the spike frequency vs injected current curve) in $\mathrm{GFP}^{+}$cells compared with neighboring GFP $^{-}$neurons (Fig. 1c-e) (two-way repeated measures ANOVA with injected current $\times$ cell: $F_{6,90}=4.55, p<0.05$ ). The average firing rate of $\mathrm{GFP}^{+}$and $\mathrm{GFP}^{-}$neurons with current injections between 100 and $150 \mathrm{pA}$ demonstrates the distribution $\left(t_{(15)}=\right.$ $4.07, p=0.001$ ). This increase was not accompanied by a change in the resting membrane potential (Figs. 1f) $(-63.8 \pm 1.2 \mathrm{mV}$ for $\mathrm{GFP}^{-}$and $64.1 \pm 1.0 \mathrm{mV}$ for $\mathrm{GFP}^{+}, p>0.05$ ). Therefore, as predicted from previous work, hippocampus-dependent learning increased the excitability of CA1 neurons for several days.

Relearning activates excitable hippocampal neurons

Once CA1 neurons become excitable, we predict they are reactivated when the animal learns something new. To test this idea, we fear conditioned mice in context $A$ followed 2 days later by training in context $B(A B)$. Excitable cells were labeled with $\mathrm{H} 2 \mathrm{~B}-\mathrm{GFP}$ during learning in $\mathrm{A}$ and activity in $\mathrm{B}$ was indexed via endogenous C-Fos expression. Reactivation was calculated as the percentage of $\mathrm{H}_{2} \mathrm{~B}-\mathrm{GFP}^{+}$cells that co-expressed c-Fos. For comparison, we trained two additional groups of mice. The first was fear conditioned in context $A$ twice $(A A)$ and the second remained in the homecage with DOX removed for a 2-day period
A.

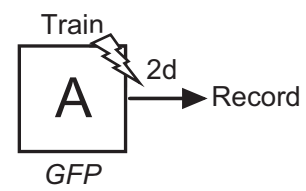

C.

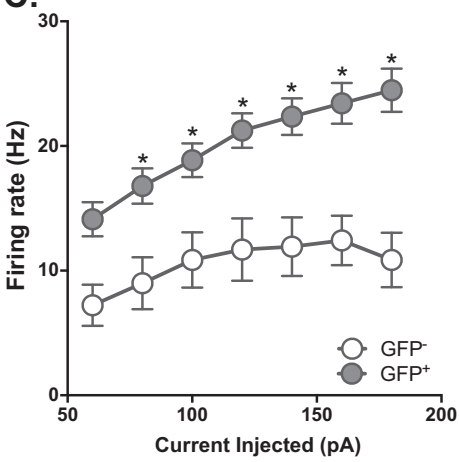

E.

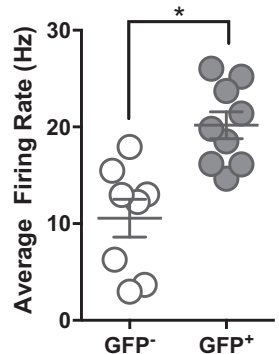

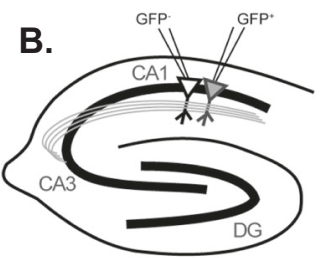

D.

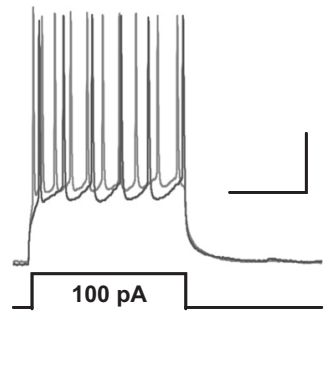

Fig. 1 Hippocampal neurons become more excitable after context fear learning. a Experimental design. Animals were trained and active neurons tagged with GFP in context $A$ and then hippocampal slices were made 2 days later. $\mathbf{b}$ Whole-cell patch clamp recordings were obtained from $\mathrm{GFP}^{+}$and neighboring $\mathrm{GFP}^{-} \mathrm{CA} 1$ pyramidal neurons. c Action potential firing rates evoked by depolarizing current injection from $\mathrm{GFP}^{+}$and $\mathrm{GFP}^{-}$CA1 pyramidal neurons recorded from TetTag mice after fear conditioning. Data represent mean \pm SEM. A two-way repeated measures ANOVA (group $\times$ current step) had a significant effect of group. The GFP ${ }^{+}$group ( $n=9$ cells, 4 animals) had a higher firing rate per current injection than the $\mathrm{GFP}^{-}$group ( $n=8$ cells, 5 animals). d Sample traces from GFP ${ }^{+}$ (gray) and $\mathrm{GFP}^{-}$(black) neurons with a $100 \mathrm{pA}$ current injection (scale bars: $250 \mathrm{~ms}, 20 \mathrm{mV}$ ). e Average firing rates for each cell from $100-150 \mathrm{pA}$ of injected current. GFP ${ }^{+}$cells (mean, $20.2 \pm 1.4 \mathrm{~Hz}$ ) had significantly higher firing rates than $\mathrm{GFP}^{-}$neurons (mean, 10.6 \pm 2.0 $\mathrm{Hz}$ ). $\mathbf{f}$ Resting membrane potential is unchanged between $\mathrm{GFP}^{+}$ (mean, $-64.1 \pm 1.0 \mathrm{mV}$ ) and $\mathrm{GFP}^{-}$(mean, $-63.8 \pm 1.2 \mathrm{mV}$ ) neurons. Error bars represent SEM. Significance values were set at $p<0.05\left(^{*}\right)$

(control) (Fig. 2a). As expected, mice retrained in context B froze less during the baseline period than those retrained in context $A$ $\left(t_{(6)}=2.48, p<0.05\right)$. This result indicates that fear of $A$ does not generalize to context $B$. However, after the shocks were administered, mice froze an equivalent amount in both environments (Fig. 2b) $\left(t_{(6)}=0.35, p>0.05\right)$. Ninety minutes after the second training session, mice were sacrificed and immunohistochemistry for c-Fos was performed.

As expected, the number of GFP ${ }^{+} \mathrm{CA} 1$ neurons was significantly higher in fear conditioned mice compared to homecage controls (one-way ANOVA, significant effect of treatment $F(2,10)=13.18$, $p<0.05$, post-hoc comparisons, A vs. control, $p<0.05$, B vs. control, $p<0.05$, A vs. B, $p>0.05$ ) (Fig. $2 \mathrm{c}$ ). A similar pattern was observed for c-Fos expression (one-way ANOVA, significant effect of treatment $F_{(2,10)}=123.6 p<0.05$, post-hoc comparisons, A vs. control, $p<0.05$, B vs. control $p<0.05$, test $A$ vs. $B, p=0.04$ ) 
A.
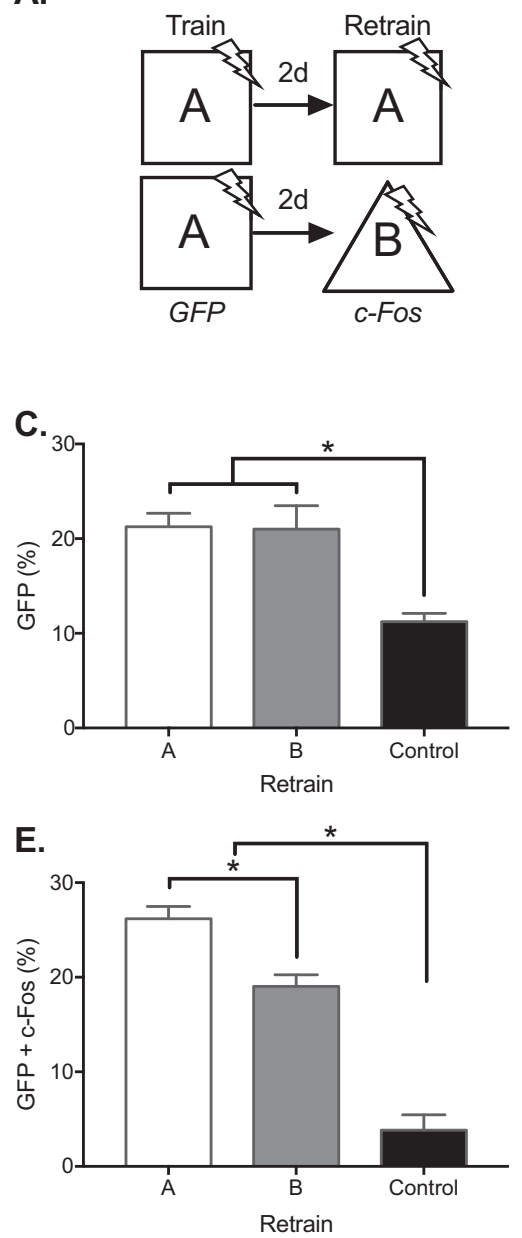

B.
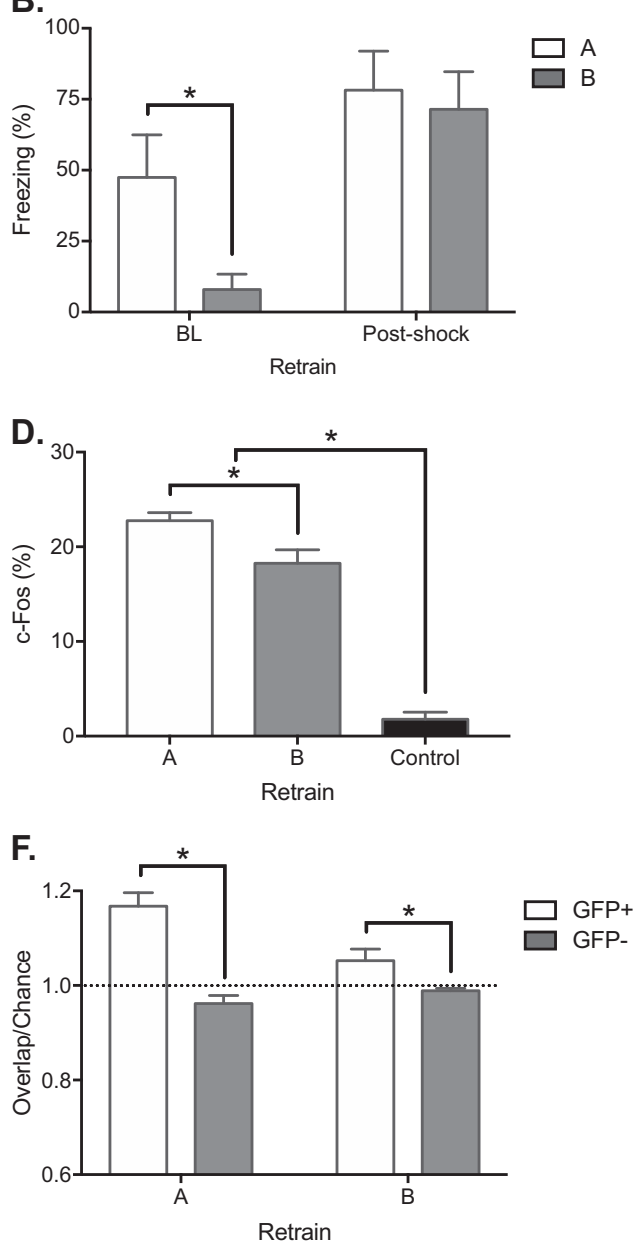

Fig. 2 Relearning activates excitable hippocampal neurons. a Experimental design. Animals were trained and active neurons tagged with GFP in context $A$, then retrained in $A$ or $B(n=4,4)$, and tissue stained for c-Fos expression. b Percent freezing in the re-training context (white: $A$, gray: $\mathrm{B})$ during the 3-min baseline $(\mathrm{BL})$ and the final minute post-shock. Low baseline freezing in context $\mathrm{B}$ indicates that mice were able to discriminate between contexts. c Percent GFP expressed during tagging in context A or homecage (control, $n=5$ ). Animals trained in context $A$ (and later retrained in A and B) express higher GFP than animals exposed to the homecage. $\mathbf{d}$ Percent c-Fos expressed during re-training in context A, B, or homecage (control). Animals retrained in context A or B express higher c-Fos than control animals. e Reactivation of neurons when retrained in A (white), B (gray), or no re-training (black), as measured by the percent of GFP neurons expressing c-Fos. Reactivation in $A$ was significantly higher than reactivation in $B$, and reactivation in $A$ or $B$ was significantly higher than reactivation with no re-training. f Activation of $\mathrm{GFP}^{+}$and $\mathrm{GFP}^{-}$neurons during re-training. Overlap between $\mathrm{GFP}^{+}$or $\mathrm{GFP}^{-}$cells was normalized to that expected by chance. For $\mathrm{GFP}^{+}$neurons this calculation was $\left(\mathrm{c}-\right.$ Fos in GFP $\left.{ }^{+}\right) \div\left(\mathrm{c}-\mathrm{Fos} \times \mathrm{GFP}^{+}\right)$and for GFP ${ }^{-}$neurons it was $\left(\mathrm{c}-\mathrm{Fos}_{\text {in }} \mathrm{GFP}^{-}\right) \div\left(\mathrm{c}-\mathrm{Fos} \times \mathrm{GFP}^{-}\right)$. Overlap between $\mathrm{GFP}^{+}$cells and c-Fos was significantly greater than the overlap between GFP ${ }^{-}$cells and c-Fos. This was true for mice retrained in context A or B. Error bars represent SEM. Significance values were set at $p<0.05\left({ }^{*}\right)$

(Fig. 2d). Consistent with previous work, CA1 neurons were more likely to be reactivated (i.e. co-express GFP and c-Fos) in the AA group compared to the $A B$ group $\left(t_{(6)}=3.93, p<0.05\right)[9,23]$. Nonetheless, the percentage of reactivated neurons was quite similar whether the mice were retrained in $A(26 \%)$ or $B(19 \%)$. In addition, reactivation in context $A\left(t_{(7)}=10.24, p<0.05\right)$ and context $\mathrm{B}\left(t_{(7)}=7.07, p<0.05\right)$ were both significantly higher than that observed in the homecage control group (4\%), which did not undergo training or testing (Fig. 2e).

To determine if $\mathrm{GFP}^{+}$cells were biased to be activated during re-training, we normalized overlap to the total amount of c-Fos and GFP in CA1 $[9,21]$. For GFP ${ }^{+}$neurons this calculation was (cFos in $\left.\mathrm{GFP}^{+}\right) \div\left(\mathrm{c}-\mathrm{Fos} \times \mathrm{GFP}^{+}\right)$. For $\mathrm{GFP}^{-}$neurons this calculation was (c-Fos in $\left.\mathrm{GFP}^{-}\right) \div\left(\mathrm{c}-\mathrm{Fos} \times \mathrm{GFP}^{-}\right.$). Positive values indicate that the amount of overlap is greater than that expected by chance (Fig. 2f). Similar to our published work, we found that overlap between $\mathrm{GFP}^{+}$cells and c-Fos was significantly greater than the overlap between $\mathrm{GFP}^{-}$cells and c-Fos. This was true in both the
AA $(t(3)=4.96, p<0.05)$ and $A B$ groups $(t(3)=3.24, p<0.05)$. These results suggest that there is a bias for $\mathrm{GFP}^{+}$cells to be activated during re-training in context $A$ and context $B$.

Initial learning requires NMDARs but relearning does not We hypothesize that excitable hippocampal neurons are able to encode new information without NMDARs. To test this idea, we fear conditioned mice in context A followed 2 days later by retraining in context $B$. One group received APV infusions into the dorsal hippocampus before learning in A but no infusions prior to training in $B$. A second group received no infusions before training in $A$ and $A P V$ infusions prior to training in B. Memory was assessed 1 day after each training session by placing the mice back into the context and measuring freezing [24]. Consistent with previous work, mice that received APV prior to training in context A showed impaired memory in that environment relative to saline controls (two-way repeated measures ANOVA, drug effect, $\mathrm{F}_{(1,15)}=8.76$, $p<0.05)$. When these same animals were subsequently trained in 
A.

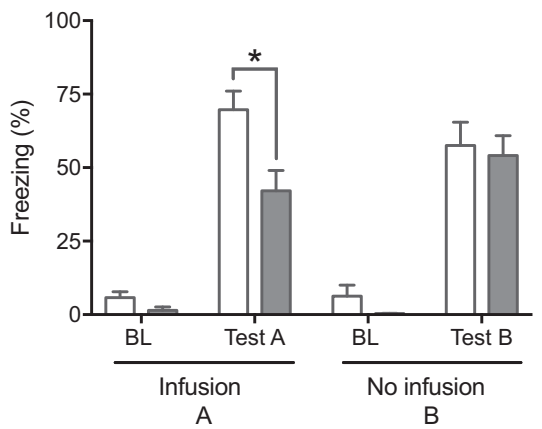

C.

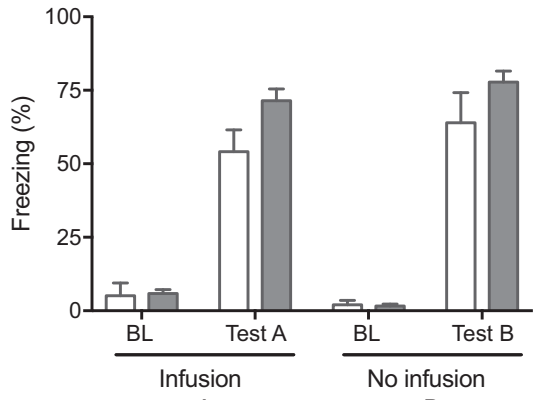

B.

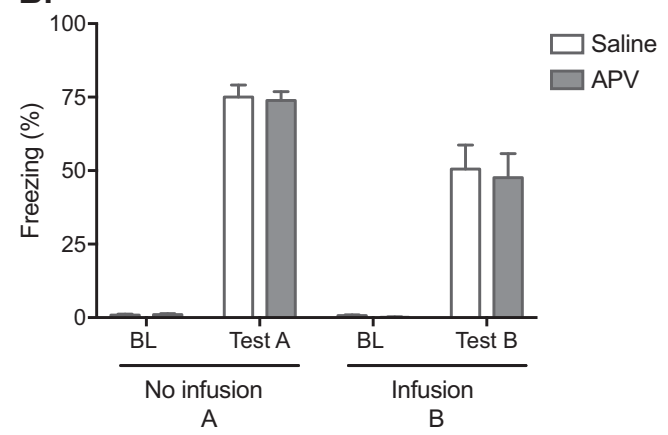

D.

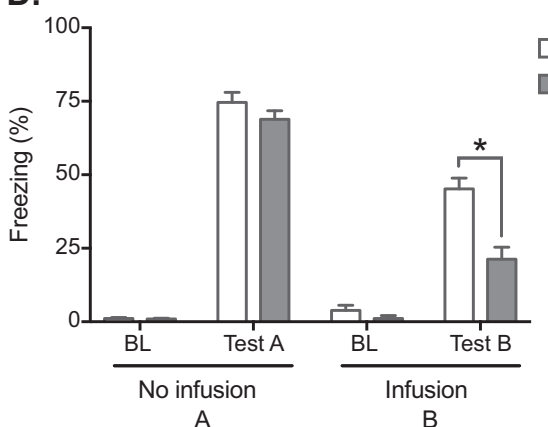

Fig. 3 Initial learning requires NMDARs while subsequent learning is mediated by mGluRs. Mice were fear conditioned in context A followed by training in context B 3 days later. The NMDAR antagonist APV or the group I/II mGluR antagonist MCPG was infused into the dorsal hippocampus 15 min prior to training in context A or context B. a APV impaired learning when infused prior to training in context A and no subsequent deficit was observed when these mice were trained in context $\mathrm{B}$ ( $n=8$ saline, 9 APV). $\mathbf{b}$ APV infusion prior to training in context B had no effect in memory encoding ( $n=8$ saline, 9 APV). c MCPG did not cause a memory deficit when infused prior to training in context A and mice were able to learn subsequent conditioning in context $B$ ( $n=5$ saline, 5 MCPG). d Mice previously conditioned in context $A$ demonstrated a memory impairment when MCPG was infused preceding training in context B ( $n=14$ saline, 7 MCPG). Low baseline freezing levels in context B indicate that mice were able to discriminate between contexts. Error bars represent SEM. Significance values were set at $p<$ $0.05(*)$

context $B$ with no infusions, memory was similar in both groups (two-way repeated measures ANOVA, $\mathrm{F}_{(1,15)}=0.63, p>0.05$ ) (Fig. 3a). Separate groups of mice were trained in context $A$ with no infusions. Not surprisingly, these animals showed equivalent levels of freezing in this environment (two-way repeated measures ANOVA, $\left.F_{(1,15)}=0.04, p>0.05\right)$. When the same mice were subsequently trained in context $B$, infusions of APV did not impair memory (two-way repeated measures ANOVA, $\mathrm{F}_{(1,15)}=0.08, p>$ $0.05)$ (Fig. 3b). This result demonstrates that relearning can occur in the absence of NMDAR activation. Importantly, as in our reactivation experiment, mice did not simply generalize their fear across contexts. In both groups, freezing levels were low in context $B$ during the baseline period and increased after the shocks were administered (two-way repeated measures ANOVA, period effect, $\left.F_{(1,15)}=71.92, p<0.05\right)$.

NMDAR-independent learning is mediated by mGluRs

Previous studies found that mGluRs can mediate LTP when NMDARs are blocked $[17,25]$. In the hippocampus, this type of plasticity is observed when cells are strongly excited [26]. One possibility, therefore, is that excitable neurons reactivated in context $B$ undergo synaptic strengthening via mGluR activation. To test this idea, mice underwent the same protocol as in the previous experiment except that the mGluR antagonist MCPG was infused into the hippocampus before training in context $A$ or context $B$. Infusions of MCPG prior to learning in A had no effect on memory (two-way repeated measures ANOVA, $\mathrm{F}_{(1,8)}=2.812, p>$ $0.05)$. This suggests that mGluR activation is not required for initial context learning. When the same mice were trained in context B with no infusions, memory was also similar (two-way repeated measures ANOVA, $\mathrm{F}_{(1,8)}=1.306, p>0.05$ ) (Fig. 3c). To determine if mGluRs are required for relearning, separate animals were trained in context $A$ with no infusions and then given infusions of MCPG prior to conditioning in context B. Memory for A (where no infusions were given) was similar in both groups (two-way repeated measures ANOVA, $\left.F_{(1,19)}=1.267, p>0.05\right)$. However, when the same mice were retrained in context $B$, blockade of mGluRs with MCPG significantly impaired memory (two-way repeated measures ANOVA, drug effect, $\left.\mathrm{F}_{(1,19)}=12.29, p<0.05\right)$ (Fig. 3d). Together, our data suggest that initial learning requires NMDARs while relearning is dependent on the activation of mGluRs.

\section{DISCUSSION}

The biological basis of memory has been thoroughly characterized in the hippocampus $[27,28]$. It is thought that memories are formed via activation of post-synaptic NMDARs, which increase calcium influx and initiate intracellular events that lead to gene expression and synaptic strengthening. Our data suggest this model is incomplete. NMDARs are not always required for memory storage; they are only needed when animals have no prior experience with the behavioral task. If prior training is given, then new spatial and contextual memories can be formed in the presence of NMDAR antagonists $[6,7,9,11]$. It should be noted that for NMDAR-independent learning to occur, prior training has to be on the same (or a similar) task. If animals are trained in the water maze, for example, subsequent context fear learning still requires NMDAR activation [11].

To conclude that hippocampal NMDARs are not required for memory, several conditions must be met. First, animals have to 
form a new memory during the training session and not simply generalize from previous experiences. This was true in the current study as baseline freezing in context B (a measure of generalization) was very low. Mice froze significantly more in context $A$, which indicates that they could distinguish between the conditioning environments (Fig. 2b). Similarly, in the Morris water maze, experienced animals are able to form new spatial memories in the presence of NMDAR antagonists even when the room, platform location, and distal cues are altered [6]. A second condition is that NMDAR-independent memories must depend on the hippocampus. If the second learning event is encoded by a different brain region, then blocking NMDARs in the hippocampus tells you nothing about their requirement for memory. To address this possibility, lesions of the hippocampus were made following fear conditioning in two different contexts or after animals learned to navigate in two distinct watermazes. In each case, NMDAR-dependent and NMDAR-independent memories were equally impaired by hippocampus damage [6, 9]. These data suggest that prior experience alters plasticity such that new memories can be formed in the absence of NMDAR activation. A change in the mechanisms of plasticity that results from experience is often referred to as metaplasticity.

Changes in the plasticity mechanisms underlying memory are not unique to the hippocampus or spatial/contextual learning. Similar effects have been observed in the piriform cortex during an olfactory discrimination task. In this paradigm, the encoding of novel odor-reward pairs initially requires NMDAR activation. However, after several days of training, rats can learn new odor-reward pairs in the presence of NMDAR antagonists. This shift in plasticity is accompanied by an increase in the intrinsic excitability of neurons in the piriform cortex and a change in postsynaptic NMDAR subunit composition (increased GluN2A and reduced GluN2B) [7]. Based on these data, we hypothesized that NMDAR-independent context fear conditioning would also be correlated with an increase in the intrinsic excitability of hippocampal neurons. To test this idea, we labeled active CA1 neurons during conditioning with the long-lasting fluorescent protein H2B-GFP. Two days later, whole-cell recordings were conducted and revealed that the intrinsic excitability of tagged neurons was significantly elevated compared to non-labeled cells (Fig. 1a). In addition, a large number of excitable CA1 neurons (19\%) were reactivated when the mice were trained in a new context. This number was only slightly lower than that observed when mice were trained in the same context twice $(26 \%)$ and significantly higher than reactivation in homecage controls (4\%) (Fig. 2c). We suggest, therefore, that NMDAR-independent learning is mediated by the activation of excitable hippocampal neurons.

If our hypothesis is correct, then blocking increases in excitability or preventing the reactivation of excitable cells should reduce NMDAR-independent learning. Alternatively, if increases in excitability are transient, then re-training could be examined after cellular activity returns to baseline. After trace eyeblink conditioning, CA 1 and CA3 neurons become more excitable and return to baseline within a week [14, 29]. Similarly, in an olfactory discrimination task, neuronal excitability in the piriform cortex is increased for several days before returning to baseline. During this period of enhanced excitability, animals are able to learn new odor pairs in the presence of NMDAR antagonists [13]. Based on these results, we predict that increased excitability after context fear conditioning will also be transient. If so, NMDAR-independent learning should disappear over time. Consistent with this idea, a recent study found that NMDARs are required for both learning (in context A) and relearning (in context B) if the fear conditioning sessions are separated by several weeks [30].

The finding that plasticity is induced by NMDARs and refined by other mechanisms has also been observed in the barrel cortex. In this case, activation of NMDARs is required to initiate plasticity following whisker trimming, but not for additional changes in synaptic strength that occur hours to days later. Instead, these latter changes are mediated by the activation of mGluRs [25]. If mice are trained on a whisker discrimination task during this latter phase, learning is blocked by the administration of an mGluR antagonist but is not affected by an NMDAR antagonist. We determined if a similar process occurs in the hippocampus after context fear learning. As expected, context conditioning in naïve animals was blocked by the NMDAR antagonist APV. However, if the mice were trained in two contexts, learning about the second environment was not affected by APV but instead by infusions of the mGluR antagonist MCPG. This result suggests that metaplastic changes in the hippocampus (induced by learning) may be similar to those observed in the barrel cortex. Follow-up experiments are needed to determine if the induction of LTP on excitable hippocampal neurons is also dependent on mGluRs but not NMDARs.

In hippocampal slices, robust stimulation $(200 \mathrm{~Hz})$ induces a form of LTP that is independent of NMDARs and dependent on the activation of mGluRs [17]. This form of plasticity is thought to be engaged when cells are firing at high frequencies and NMDAR activity (or plasticity) is saturated. Both of these conditions likely exist during relearning. After context fear conditioning, synaptic connections between co-active neurons (identified via c-Fos expression) are strengthened and further LTP is occluded [31]. Given that many of these potentiated cells are activated during relearning (Fig. 2e) additional synaptic strengthening may not be possible via NMDAR activation. Instead, engagement of mGluRs may be required for new learning to occur. The increased excitability of CA1 neurons could facilitate this process by enhancing the firing rate of previously active cells. The saturation of NMDAR-mediated LTP may result from metaplastic increases in the synaptic GluN2A/GluN2B ratio that increases the threshold for additional LTP [7, 32-34]. As the threshold for NMDAR-mediated LTP increases, additional forms of synaptic plasticity may be gated, including mGluR-based mechanisms. Indeed, at the largest dendritic spines, mGluR signaling is required for structural plasticity [37].

Finally, it should be mentioned that context fear memories can also be formed in the ventral hippocampus [35]. It is possible, therefore, that animals use this mechanism to learn when APV is infused into the dorsal hippocampus. However, we and others have shown that systemic injections of NMDAR antagonists (which affect the entire hippocampus) do not prevent context fear learning in pre-trained animals [9-11]. Moreover, selective lesions of the dorsal hippocampus produce dense amnesia for both NMDAR-dependent and NMDAR-independent context fear memories [9]. In addition, the current MCPG data indicate that plasticity mechanisms in the dorsal hippocampus are engaged during NMDAR-independent learning and are required for memory formation. Nonetheless, future studies will need to look closely at the contribution of the ventral hippocampus and clarify the plasticity mechanisms that are required in that region.

In summary, our data suggest that NMDARs are not always required for memory formation. These receptors appear to be selectively engaged when an animal learns a behavioral task for the first time. Once learning has occurred, new memories can be formed on the same task (or a similar one) via the activation of mGluRs. We hypothesize that mGluR-mediated plasticity occurs on excitable hippocampal neurons that were active during the original learning experience.

\section{FUNDING:}

These experiments were supported by a Whitehall Foundation Research Grant to B.J. W. and a CNPq/Brazil graduate fellowship to A.P.C. "SWE Process: 202250/2015-6". 


\section{ADDITIONAL INFORMATION}

Competing interests: The authors declare no competing interests.

Publisher's note: Springer Nature remains neutral with regard to jurisdictional claims in published maps and institutional affiliations.

\section{REFERENCES}

1. Bouton ME. Context, time, and memory retrieval in the interference paradigms of pavlovian learning. Psychol Bull. 1993;114:80-99.

2. Winocur G. The effects of retroactive and proactive interference on learning and memory in old and young rats. Dev Psychobiol. 1984;17:537-45.

3. Tse D, Langston RF, Kakeyama M, Bethus I, Spooner PA, Wood ER, et al. Schemas and memory consolidation. Science. 2007;316:76-82.

4. Tse D, Takeuchi T, Kakeyama M, Kajii Y, Okuno H, Tohyama C, et al. Schemadependent gene activation and memory encoding in neocortex. Science. 2011;333:891-5

5. Saucier D, Cain DP. Spatial learning without NMDA receptor-dependent longterm potentiation. Nature. 1995;378:186-9.

6. Bannerman DM, Good MA, Butcher SP, Ramsay M, Morris RGM. Distinct components of spatial learning revealed by prior training and NMDA receptor blockade. Nature. 1995;378:182-6.

7. Quinlan EM, Lebel D, Brosh I, Barkai E. A molecular mechanism for stabilization of learning-induced synaptic modifications. Neuron. 2004;41:185-92.

8. Sanders MJ, Fanselow MS. Pre-training prevents context fear conditioning deficits produced by hippocampal NMDA receptor blockade. Neurobiol Learn Mem. 2003:80:123-9.

9. Tayler KK, Lowry E, Tanaka K, Levy B, Reijmers L, Mayford M, et al. Characterization of NMDAR-independent learning in the hippocampus. Front Behav Neurosci. 2011;5:1-12.

10. Wiltgen BJ, Royle GA, Gray EE, Abdipranoto A, Thangthaeng N, Jacobs N, et al. A role for calcium-permeable AMPA receptors in synaptic plasticity and learning. PLOS ONE. 2010;4:158,1-11.

11. Wiltgen BJ, Wood AN, Levy B. The cellular mechanisms of memory are modified by experience. Learn Mem. 2011;18:747-50.

12. Cai DJ, Aharoni D, Shuman T, Shobe J, Biane J, Song W, et al. A shared neural ensemble links distinct contextual memories encoded close in time. Nature. 2016;534:115-8.

13. Chandra N, Barkai E. A non-synaptic mechanism of complex learning: modulation of intrinsic neuronal excitability. Neurobiol Learn Mem. 2017. https://doi.org/ 10.1016/j.nlm.2017.11.015.

14. Moyer JR, Thompson LT, Disterhoft JF. Trace eyeblink conditioning increases CA1 excitability in a transient and learning-specific manner. J Neurosci. 1996;16:5536-46.

15. Saar D, Barkai E. Long-term modifications in intrinsic neuronal properties and rule learning in rats. Eur J Neurosci. 2003;17:2727-34.

16. Nicoll RA, Schmitz D. Synaptic plasticity at hippocampal mossy fibre synapses. Nat Rev Neurosci. 2005;6:863-76.

17. Wang H, Ardiles AO, Yang S, Tran T, Posada-Duque R, Valdivia G, et al. Metabotropic glutamate receptors induce a form of LTP controlled by translation and Arc signaling in the hippocampus. J Neurosci. 2016;36:1723-9.
18. Reijmers LG, Mayford M. Genetic control of active neural circuits. Front Mol Neurosci. 2009;2:1-8.

19. Ting JT, Daigle TL, Chen Q, Feng G. Acute brain slice methods for adult and aging animals: application of targeted patch clamp analysis and optogenetics. Methods Mol Biol. 2014;1183:221-42.

20. Nakazawa Y, Pevzner A, Tanaka KZ, Wiltgen BJ. Memory retrieval along the proximodistal axis of CA1. Hippocampus. 2016;26:1140-8.

21. Tanaka KZ, Pevzner A, Hamidi AB, Nakazawa Y, Graham J, Wiltgen BJ. Cortical representations are reinstated by the hippocampus during memory retrieval. Neuron. 2014;84:347-54.

22. Dong HW. The Allen reference atlas: a digital color brain atlas of the C57BI/6J male mouse. Hoboken, NJ: USJohn Wiley Sons Inc.; 2008.

23. Ramirez S, Liu X, Lin P-A, Suh J, Pignatelli M, Redondo RL, et al. Creating a false memory in the hippocampus. Science. 2013;341:387-91.

24. Anagnostaras SG. Automated assessment of Pavlovian conditioned freezing and shock reactivity in mice using the VideoFreeze system. Front Behav Neurosci. 2010;5-e12818,1-18.

25. Clem RL, Celike T, Barth AL. Ongoing in vivo experience triggers synaptic metaplasticity in the neocortex. Science. 2008;319:101-4.

26. Wang $\mathrm{S}-\mathrm{H}$, Redondo $\mathrm{RL}$, Morris RGM. Relevance of synaptic tagging and capture to the persistence of long-term potentiation and everyday spatial memory. Proc Natl Acad Sci USA. 2010;107:19537-42.

27. Elgersma $Y$, Silva aJ. Molecular mechanisms of synaptic plasticity and memory. Curr Opin Neurobiol. 1999;9:209-13.

28. Morris RGM, Davis S, Butcher SP. Hippocampal synaptic plasticity and NMDA receptors: a role in information storage? Philos Trans $\mathrm{R}$ Soc $\mathrm{B}$ Biol Sci. 1990;329:187-204

29. Thompson LT, Moyer JR, Disterhoft JF. Transient changes in excitability of rabbit CA3 neurons with a time course appropriate to support memory consolidation. J Neurophysiol. 1996;76:1836-49.

30. Crestani AP,Sierra RO,Machado A,Haubrich J,Scienza KM,de Oliveira Alvares L, et al. Hippocampal plasticity mechanisms mediating experience-dependent learning change over time. Neurobiol Learn Mem. 2018;150:56-63.

31. Choi J-H, Sim S-E, Kim J-I, Choi DIl, Oh J, Ye S, et al. Interregional synaptic maps among engram cells underlie memory formation. Science. 2018;360:430-5.

32. Gray JA, Shi Y, Usui H, During MJ, Sakimura K, Nicoll RA. Distinct modes of AMPA receptor suppression at developing synapses by GluN2A and GluN2B: single-cell NMDA receptor subunit deletion in vivo. Neuron. 2011;71:1085-101.

33. Philpot BD, Cho KKA, Bear MF. Obligatory role of NR2A for metaplasticity in visual cortex. Neuron. 2007;53:495-502.

34. Quinlan EM, Philpot BD, Huganir RL, Bear MF. Rapid, experience-dependent expression of synaptic NMDA receptors in visual cortex in vivo. Nat Neurosci. 1999;2:352-7.

35. Wang SH, Finnie PSB, Hardt O, Nader K. Dorsal hippocampus is necessary for novel learning but sufficient for subsequent similar learning. Hippocampus. 2012;22:2157-70.

36. Franklin KBJ, Paxinos G. The mouse brain in stereotaxic coordinates. Amsterdam: Elsevier Academic Press; 2008.

37. Oh WC, Hill TC, Zito K. Synapse-specific and size-dependent mechanisms of spine structural plasticity accompanying synaptic weakening. Proc Natl Acad Sci U S A. 2013;110:E305-E312. 